\title{
Electrochemical Detection of Sudan I Using a Multi-Walled Carbon Nanotube/Chitosan Composite Modified Glassy Carbon Electrode
}

\author{
Min Wu, Wanrong Tang, Junlin Gu, Qingjiang Wang*, Pingang He, Yuzhi Fang \\ Department of Chemistry, East China Normal University, Shanghai, China \\ Email: *qjwang@chem.ecnu.edu.cn
}

Received March 8, 2013; revised April 21, 2013; accepted May 4, 2013

Copyright (C) 2013 Min Wu et al. This is an open access article distributed under the Creative Commons Attribution License, which permits unrestricted use, distribution, and reproduction in any medium, provided the original work is properly cited.

\begin{abstract}
In this work, a simple and sensitive electrochemical method was developed to determine Sudan I by cyclic voltammetry and differential pulse voltammetry using a glassy carbon electrode modified with a chitosan/carbon nanotube composite. In cyclic voltammetry, Sudan I exhibited a well-defined oxidation peak located at $0.72 \mathrm{~V}$ at the multi-walled carbon nanotube (MWCNT)/chitosan-modified GCE. The determination conditions, including $\mathrm{pH}$, scan rate, and chitosan: MWCNT mass ratio at the modified electrode, were optimized. Under the optimum experimental conditions, Sudan I could be linearly detected by differential pulse voltammetry with a detection limit of $3.0 \times 10^{-8} \mathrm{~mol} \cdot \mathrm{L}^{-1}$.
\end{abstract}

Keywords: Sudan I; Electrochemical Detection; Chitosan; Multi-Walled Carbon Nanotubes

\section{Introduction}

Sudan I (1-[(2,4-dimethylphenyl)azo]-2-naphthalenol) is a synthetic azo-colorant that has been widely used in various industrial and scientific applications, such as in waxes and textile colorants, shoe polishes and food additives. Sudan I has been reported to cause tumors in mouse liver and bladder and is a possible carcinogen and mutagen in humans [1]. Hence, Sudan I is classified as a category 3 carcinogen by the International Agency for Research on Cancer (IARC) and its addition to food products is forbidden in most countries. Although Sudan I concentrations of $100-1000 \mathrm{mg} \cdot \mathrm{kg}^{-1}$ are required to produce the color of chili products, commonly reported levels of Sudan dyes are in the low $\mathrm{mg} \mathrm{kg}^{-1}$ range. Therefore, the sensitive and rapid determination of low levels of Sudan I in food is of enormous importance [2].

To date, many methods have been investigated for the determination of Sudan I, such as high-performance liquid chromatography (HPLC) [3-9], gas chromatography (GC) [10], capillary electrophoresis [11], immunoanalysis $[12,13]$, chemiluminescence flow injection analysis [14], and plasmon resonance light scattering (PRLS) [15]. Most of these techniques exhibit high sensitivity and excellent selectivity, but some are also time-consuming, expensive, and unsuitable for in situ assays.

${ }^{*}$ Corresponding author.
Electrochemical methods have also been used to determine Sudan I due to the advantages such as fast response, high sensitivity, favorable portability, and low cost. For instance, in a previous study, Sudan I was determined based on its reduction at an electrochemically activated glassy carbon electrode [16]. To improve the detection sensitivity of Sudan I, many types of chemically modified electrodes have been investigated, especially those incorporating carbon nanomaterials. Carbon nanotubes (CNTs) include single-walled carbon nanotubes (SWCNTs) and multi-walled carbon nanotubes (MWCNTs). CNTs are porous carbon nanostructures, possessing properties such as high electrical conductivity, a high surface area, chemical stability, and significant mechanical strength; therefore, they can be used to promote electron transfer reactions when used as electrode materials in electrochemical devices [17-21]. Glass carbon electrodes modified by single-walled carbon nanotubes and iron (III) porphyrin have been used in square wave voltammetry (SWV) to determine Sudan I [22]. Huanshun Yin et al. reported the electrochemical behavior of Sudan I at glassy carbon electrodes modified with $\mathrm{Fe}_{3} \mathrm{O}_{4}$ nanoparticles and its determination in food samples [23].

To provide CNT-modified electrodes with sufficient stability, polymers have been studied as conductive adhesives in chemically modified electrodes. Chitosan (CHIT) 
is a natural polymer with attractive properties, such as biocompatibility, nontoxicity, high water permeability, high mechanical strength, and excellent film-forming abilities. Because chitosan and CNTs can form a stable complex through noncovalent binding, the stability of CNTs in aqueous chitosan solutions is greatly improved and CNTs can be uniformly distributed in a chitosan film [24-27]. A glassy carbon electrode modified with a multiwalled carbon nanotube/chitosan composite has been utilized as a new sensor for the simultaneous determination of acetaminophen and mefenamic acid in pharmaceutical preparations and biological samples [28].

Due to the clear advantages of MWCNT/chitosan composites in electrochemical analysis, in this study, we used these composites to determine Sudan I by cyclic voltammetry and differential pulse voltammetry. The determination conditions, such as $\mathrm{pH}$, scan rate, and chitosan: MWCNT mass ratio, were optimized. Under the optimum experimental conditions, Sudan I could be linearly detected by differential pulse voltammetry with a detection limit of $3.0 \times 10^{-8} \mathrm{~mol} \cdot \mathrm{L}^{-1}$. This electrochemical method was applied to determine Sudan I in chili powder samples, exhibiting high sensitivity, rapid response, and extreme simplicity.

\section{Experimental}

\subsection{Reagents and Solutions}

Sudan I (1-[(2,4-dimethylphenyl)azo]-2-naphthalenol) was purchased from Tianjin Lanlike Co. Ltd., China. A stock solution of $5.0 \times 10^{-3} \mathrm{~mol} \cdot \mathrm{L}^{-1}$ Sudan I was prepared in ethanol and stored at $4^{\circ} \mathrm{C}$ in the dark. Multi-walled carbon nanotubes (MWCNTs) (>95\% w/w, 5 - $20 \mathrm{~nm})$ were purchased from Shenzhen Nanotech Port Co., Ltd, China. The buffer solution was prepared by mixing stock solutions of $0.1 \mathrm{~mol} \cdot \mathrm{L}^{-1} \mathrm{KNO}_{3}$ and $0.05 \mathrm{~mol} \cdot \mathrm{L}^{-1} \mathrm{KH}_{2} \mathrm{PO}_{4}$ and adjusting the $\mathrm{pH}$ to 7 . All solutions were prepared with re-distilled water. All chemicals were of analytical reagent grade unless otherwise stated and used directly without further purification.

\subsection{Apparatus}

All electrochemical experiments were performed using a CHI 660C electrochemical workstation (Chenhua Instruments Co., Shanghai, China) featuring a three-electrode system, which was controlled by a personal computer. The three-electrode system was composed of a MWCNT/chitosan-modified glassy carbon electrode as the working electrode, a saturated calomel electrode (SCE) as the reference electrode, and a platinum wire as the auxiliary electrode. All electrochemical measurements were carried out at room temperature. The $\mathrm{pH}$ measurements were conducted with a pHs-3C meter (Leici Instruments Co., Shanghai, China) using a combination glass elec- trode.

\subsection{Modification of the Chemically Modified Electrodes}

A MWCNT/chitosan-modified glassy carbon electrode was fabricated as follows. First, a bare glassy carbon electrode (GCE) was polished with an alumina slurry until it was mirror-like, successively washed with re-distilled water and anhydrous alcohol in an ultrasonic bath for 3 min, and dried in air. Next, to obtain a $2 \mathrm{mg} \cdot \mathrm{mL}^{-1}$ chitosan solution, $0.05 \mathrm{~g}$ of chitosan was dispersed in $0.29 \mathrm{~mL}$ of acetic acid $\left(1.05 \mathrm{~g} \cdot \mathrm{L}^{-1}\right)$ using ultrasonic agitation $(20$ $\mathrm{min}$ ), after which the solution was collected and diluted in a $25 \mathrm{~mL}$ volumetric flask. A $0.02 \mathrm{~g}$ sample of MWCNTs was then dispersed in $10 \mathrm{~mL}$ of the chitosan solution using ultrasonic agitation (30 $\mathrm{min})$ to obtain a homogeneous mixture solution $(1: 1 \mathrm{w} / \mathrm{w})$. The prepared GCE surface was coated with $10.0 \mu \mathrm{L}$ of the resulting MWCNT/ chitosan solution, and the resultant electrode was placed in a drying oven for $15 \mathrm{~min}$ to allow the water to evaporate.

\subsection{Analytical Procedure}

The buffer solution prepared by mixing the stock solutions of $0.1 \mathrm{~mol} \cdot \mathrm{L}^{-1} \mathrm{KNO}_{3}$ and $0.05 \mathrm{~mol} \cdot \mathrm{L}^{-1} \mathrm{KH}_{2} \mathrm{PO}_{4}$ $(\mathrm{pH}=7)$ was used as the determining medium for Sudan I analysis. A $16.0 \mu \mathrm{L}$ aliquot of the Sudan I stock solution $\left(5.0 \times 10^{-3} \mathrm{~mol} \cdot \mathrm{L}^{-1}\right)$ was transferred into the electrochemical cell containing $8 \mathrm{~mL}$ of the buffer solution, after which the three-electrode system was installed. Cyclic voltammetry (CV) and differential pulse voltammetry (DPV) were carried out from 0.10 to $0.90 \mathrm{~V}$ after accumulating at $0.1 \mathrm{~V}$ for $60 \mathrm{~s}$.

\subsection{Sample Preparation}

A $1.00 \mathrm{~g}$ sample of chili powder (purchased from a local supermarket) was accurately weighed and ultrasonicated with $25 \mathrm{~mL}$ of ethanol for $30 \mathrm{~min}$. After filtration with a $0.45 \mu \mathrm{m}$ PTFE syringe filter membrane, the filtrate was collected and accurately diluted to $50 \mathrm{~mL}$ with ethanol. For electrochemical determination, $1.00 \mathrm{~mL}$ of the sample solution was diluted ten-fold by volume using the background electrolyte solution ( $\mathrm{pH} 7.0)$.

\section{Results and discussion}

\subsection{Electrochemical Behavior of Sudan I}

Figure 1 shows the chemical structure of Sudan I. The electrochemical behavior of Sudan I at the bare and MWCNT/chitosan-modified GCEs were investigated using cyclic voltammetry (CV). Figure 2 shows the cyclic voltammograms of $1.0 \times 10^{-5} \mathrm{~mol} \cdot \mathrm{L}^{-1}$ Sudan I in the buffer solution $(\mathrm{pH}=7.0)$ at the bare and the MWCNT/ 


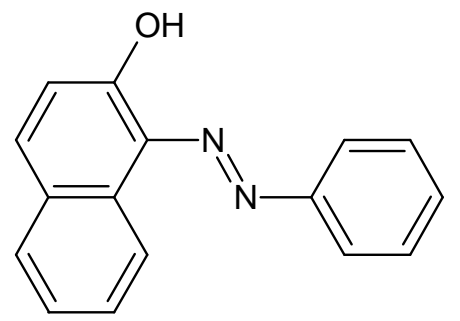

Figure 1. The chemical structure of Sudan I.

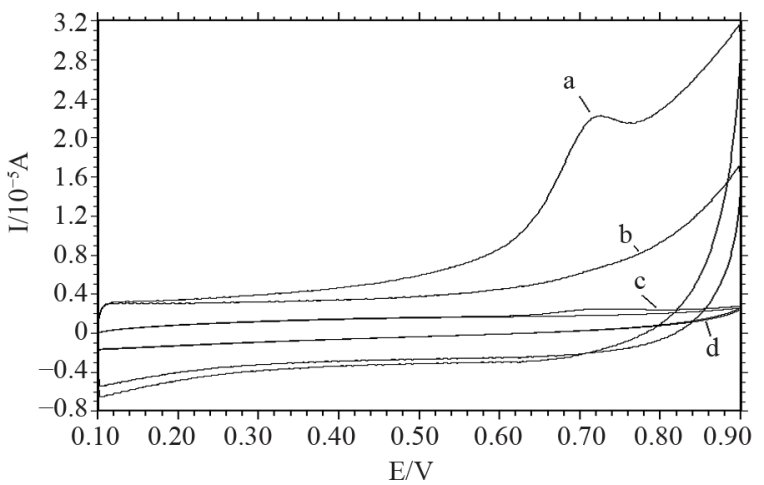

Figure 2. Cyclic voltammograms of the bare and MWCNT/ chitosan-modified GCEs. Buffer solution: $0.1 \mathrm{~mol} \cdot \mathrm{L}^{-1} \mathrm{KNO}_{3}$ and $0.05 \mathrm{~mol} \cdot \mathrm{L}^{-1} \mathrm{KH}_{2} \mathrm{PO}_{4}(\mathrm{pH}=7)$; Scan rate: $0.05 \mathrm{~V} \cdot \mathrm{s}^{-1}$.

chitosan-modified GCEs. The potential was swept from 0.10 to $0.90 \mathrm{~V}$, and the scan rate was held constant at $0.05 \mathrm{~V} \cdot \mathrm{s}^{-1}$. No redox peak was observed at either electrode (curve b, d) in the blank buffer solution. After the addition of Sudan I, a well defined oxidation peak located at $0.72 \mathrm{~V}$ was observed at the MWCNT/chitosanmodified GCE (curve a), whereas no corresponding reduction peak was observed in the reverse scan. The oxidation peak current of Sudan I at the MWCNT/chitosanmodified GCE can be attributed to the special properties of MWCNTs, such as their very large surface area, strong absorptive ability and subtle electronic properties [20]. Additionally, the chitosan that mixed with the carbon paste can also enhance the adsorption to Sudan I and promote the sensitivity of the working electrode. As a result, Sudan I was adsorbed on the surface of the electrode firstly by chitosan, and then oxidation was triggered quickly by the subtle electronic properties of MWCNTs.

\subsection{Effect of MWCNT Content in Composite}

Figure 2 clearly shows that MWCNTs can remarkably enhance the oxidation response of Sudan I. Therefore, the voltammetric response of Sudan I is strongly affected by the MWCNT content. Figure 3 shows the effect of the MWCNT content on the oxidation peak current of Sudan I $\left(1.0 \times 10^{-5} \mathrm{~mol} \cdot \mathrm{L}^{-1}\right)$ in buffer solutions $(\mathrm{pH}=7.0)$ at a scan rate of $0.05 \mathrm{~V} \cdot \mathrm{s}^{-1}$. As shown, the oxidation peak current of Sudan I increases sharply with the chitosan: MWCNT mass ratio from $1: 1$ to $1: 3$. However, the oxidation peak current clearly decreases as the mass ratio increases further. Thus, the 1:3 ratio was selected as the optimal condition.

\subsection{Effect of $\mathrm{pH}$}

According to previous reports, the oxidation peak current of Sudan I is sensitive to the $\mathrm{pH}$ of the electrolyte solution. Therefore, the effect of the $\mathrm{pH}$ level was investigated using cyclic voltammetry. Figure 4 shows the effect of $\mathrm{pH}$ on the oxidation peak current of $1.0 \times 10^{-5} \mathrm{~mol} \cdot \mathrm{L}^{-1}$ Sudan I at the MWCNT/chitosan-modified GCE. The oxidation peak currents increased substantially when the $\mathrm{pH}$ value was increased from 6.52 to 7.00 and then decreased as the $\mathrm{pH}$ was increased further from 7.00 to 7.50 . Therefore, $\mathrm{pH} 7.00$ was chosen as the optimal experimental condition in terms of sensitivity.

\subsection{Effect of Scan Rate}

The effect of the scan rate on the oxidation response of

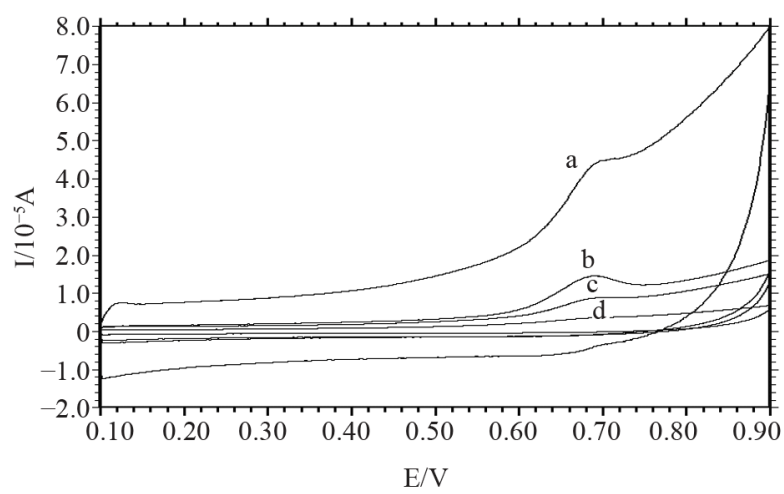

Figure 3. Cyclic voltammograms of the MWCNT/chitosanmodified GCE in pH 7.0 buffer with different chitosan. MWCNT mass ratios: (a) 1:3, (b) 1:2, (c) 1:1, (d) blank.

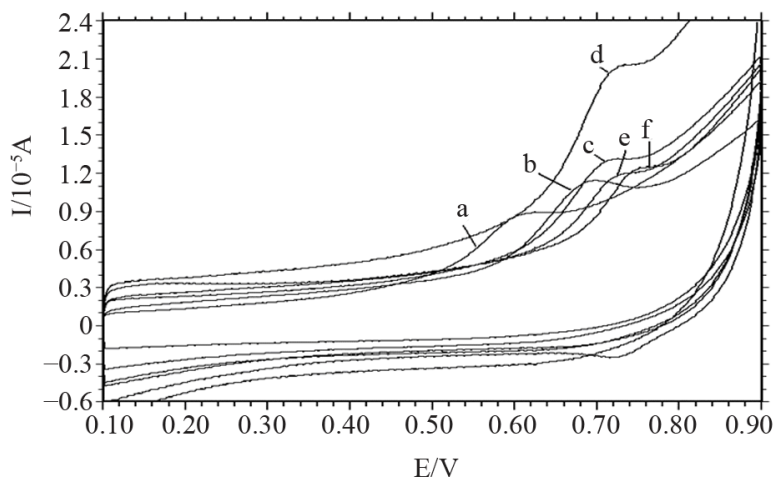

Figure 4. Cyclic voltammograms of the MWCNT/chitosanmodified GCE in $1.0 \times 10^{-5} \mathrm{~mol} \cdot \mathrm{L}^{-1}$ Sudan $\mathrm{I}$ at different $\mathrm{pH}$ levels. pH levels: (a) 6.52, (b) 6.75, (c) 6.90, (d) 7.00, (e) 7.25, (f) 7.50 . 
Sudan I was investigated by cyclic voltammetry. Figure 5 shows the cyclic voltammograms of $1.0 \times 10^{-5} \mathrm{~mol} \cdot \mathrm{L}^{-1}$ Sudan I at the MWCNT/chitosan-modified GCE at different scan rates. The oxidation peak current gradually increased with the scan rate in the range of from 0.02 to $0.4 \mathrm{~V} \cdot \mathrm{s}^{-1}$. However, a reduction peak was observed in the reverse scan when using excessively high scan rates. Ultimately, $0.05 \mathrm{~V} \cdot \mathrm{s}^{-1}$ was chosen as the optimal experimental condition.

\subsection{Calibration Graph}

To determine the linear range of this method, a calibration curve of Sudan I was constructed using differential pulse voltammetry (DPV) due to its higher sensitivity relative to that of cyclic voltammetry. Figure 6 shows the differential pulse voltammograms of the MWCNT/chitosan-modified GCE under the optimized conditions for different concentrations of Sudan I. As shown in Figure 5 , the linear range was estimated to span from $1.0 \times 10^{-7}$ to $1.0 \times 10^{-6} \mathrm{~mol} \cdot \mathrm{L}^{-1}$ and the corresponding regression

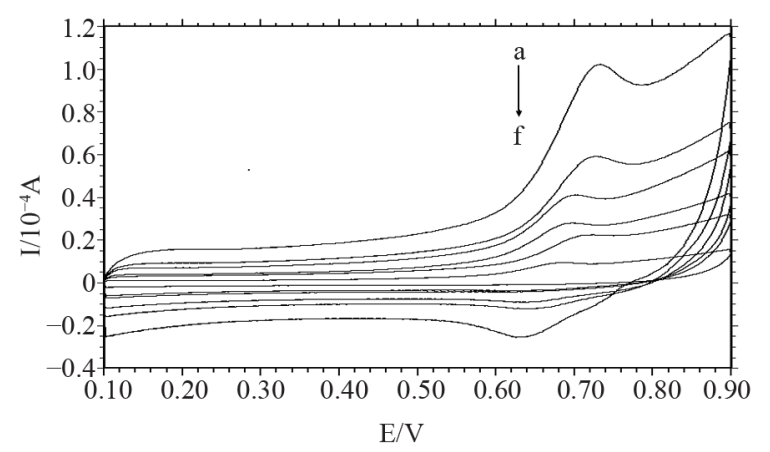

Figure 5. Cyclic voltammograms of the MWCNT/chitosanmodified GCE in buffer (pH 7.0) at different scan rates containing $1.0 \times 10^{-5} \mathrm{~mol} \cdot \mathrm{L}^{-1}$ Sudan I. Scan rates $\left(\mathrm{V} \cdot \mathrm{s}^{-1}\right)$ : (a) 0.4 ; (b) 0.3 ; (c) 0.2 ; (d) 0.1 ; (e) 0.05 ; (f) 0.02 .

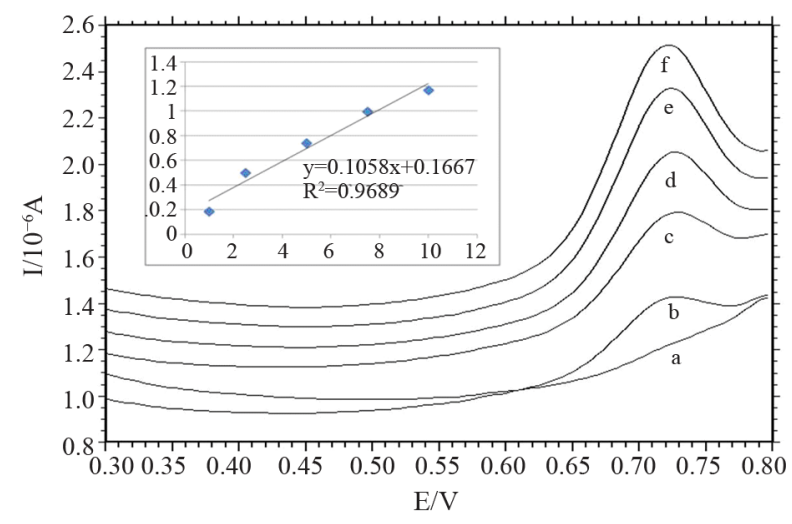

Figure 6. DPVs at the MWCNT/chitosan-modified GCE for different concentrations of Sudan $I$ in buffer solution (pH 7.0). concentrations: (a) blank, (b) $1.0 \times 10^{-7}$, (c) $2.5 \times 10^{-7}$, (d) $5.0 \times 10^{-7}$, (e) $7.5 \times 10^{-7}$, (f) $1.0 \times 10^{-6} \mathrm{~mol} \cdot \mathrm{L}^{-1}$, respectively. equation was $\mathrm{I}(\mu \mathrm{A})=0.1058 \mathrm{C}\left(10^{-7} \mathrm{~mol} \cdot \mathrm{L}^{-1}\right)+0.1667$ $(\mathrm{R} 2=0.9686)$. The limit of determination (LOD) was estimated to be $3.0 \times 10^{-8} \mathrm{~mol} \cdot \mathrm{L}^{-1}(\mathrm{~S} / \mathrm{N}=3)$. This result exemplifies the desirable properties of the MWCNT/chitosan-modified GCE.

\subsection{Analytical Application}

To verify its potential for application, the method developed in this study was applied to detect Sudan I in hot chili powder samples. A $1.00 \mathrm{~mL}$ volume of the sample stock solution was diluted ten-fold by volume using the background electrolyte solution $(\mathrm{pH} \mathrm{7.0)}$ and then subjected to DPV detection on the MWCNT/chitosan-modified GCE. After $60 \mathrm{~s}$ of accumulation at $0.1 \mathrm{~V}$, DPV was carried out from 0.10 to $0.90 \mathrm{~V}$. No Sudan I signal was observed in the differential pulse voltammograms.

To test the accuracy of this method, a recovery experiment was carried out by adding a fixed amount of Sudan I to the chili powder samples, which was then detected by DPV on the MWCNT/chitosan-modified GCE. The measurement was repeated three times for each sample, and the RSDs were ranged $90 \%$ and $97 \%$ as shown in Table 1. This satisfactory recovery demonstrates that this method is very promising for real sample analysis.

\section{Conclusion}

In this work, a simple, rapid, and sensitive electrochemical method was developed to determine Sudan I based on the highly useful properties of MWCNTs and chitosan. The fabricated electrode exhibited a good catalytic effect toward the oxidation of Sudan I, with a detection limit on the order of $10^{-8} \mathrm{~mol} \cdot \mathrm{L}^{-1}$, which is similar or lower than previously reported values obtained using electrochemical methods. Therefore, the developed method has great potential for use in the determination of Sudan I in real simples.

\section{Acknowledgements}

This work was financially supported by the program for

Table 1. Recovery of Sudan I from chili powder samples (n =3).

\begin{tabular}{ccccc}
\hline $\begin{array}{c}\text { Sample } \\
\text { No }\end{array}$ & $\begin{array}{c}\text { Spiked } \\
\left(\mu \mathrm{mol} \cdot \mathrm{L}^{-1}\right)\end{array}$ & $\begin{array}{c}\text { Found } \\
\left(\mu \mathrm{mol} \cdot \mathrm{L}^{-1}\right)\end{array}$ & $\begin{array}{c}\text { Recovery } \\
(\%)\end{array}$ & $\begin{array}{c}\text { RSD } \\
(\%)\end{array}$ \\
\hline 1 & 1.00 & 0.95 & 95 & 4.8 \\
2 & 1.00 & 0.97 & 97 & 6.2 \\
3 & 0.50 & 0.41 & 92 & 5.0 \\
4 & 0.50 & 0.43 & 96 & 4.6 \\
5 & 0.20 & 0.19 & 95 & 4.4 \\
6 & 0.20 & 0.18 & 90 & 5.4 \\
\hline
\end{tabular}


New Century Excellent Talents in University (NCET-080191) and the National Program on the Development of Scientific Instrument and Equipment (Grant 2011YQ 150072).

\section{REFERENCES}

[1] V. Martínek and M. Stiborová, "Metabolism of Carcinogenic Azo Dye Sudan I by Rat, Rabbit, Minipig and Human Hepatic Microsomes," Collection of Czechoslovak Chemical Communications, Vol. 67, No. 12, 2002, pp. 1883-1898. doi:10.1135/cccc20021883

[2] R. Rebane, I. Leito, S. Yurchenko and K. Herodes, "A Review of Analytical Techniques for Determination of Sudan I-IV Dyes in Food Matrixes," Journal of Chromatography A, Vol. 1217, No. 17, 2010, pp. 2747-2757. doi:10.1016/j.chroma.2010.02.038

[3] Y. Zhang, Y. Zhang, W. Gong, A. Gopalan and K. Lee, "Rapid Separation of Sudan Dyes by Reverse-Phase High Performance Liquid Chromatography through Statistically Designed Experiments," Journal of Chromatography A, Vol. 1098, No. 1-2, 2005, pp. 183-187. doi:10.1016/j.chroma.2005.10.024

[4] C. Yu, Q. Liu, L. Lan and B. Hu, "Comparison of Dual Solvent-Stir Bars Microextraction and U-Shaped Hollow Fiber-Liquid Phase Microextraction for the Analysis of Sudan Dyes in Food Samples by High-Performance Liquid Chromatography-Ultraviolet/Mass Spectrometry," Journal of Chromatography A, Vol. 1188, No. 2, 2008, pp. 124-131. doi:10.1016/j.chroma.2008.02.065

[5] K. Molder, A. Kunnapas, K. Herodes and I. Leito, "Fast Peaks in Chromatograms of Sudan Dyes," Journal of Chromatography A, Vol. 1160, No. 1-2, 2007, pp. 227-234. doi:10.1016/j.chroma.2007.05.052

[6] W. Liu, W. Zhao, J. Chen and M. Yang, "A Cloud Point Extraction Approach Using Triton X-100 for the Separation and Preconcentration of Sudan Dyes in Chilli Powder," Analytica Chimica Acta, Vol. 605, No. 1, 2007, pp. 41-45. doi:10.1016/j.aca.2007.10.034

[7] M. Ma, X. Luo, B. Chen, S. Su and S. Yao, "Simultaneous Determination of Water-Soluble and Fat-Soluble Synthetic Colorants in Foodstuff by High-Performance Liquid Chromatography-Diode Array Detection-Electrospray Mass Spectrometry," Journal of Chromatography A, Vol. 1103, No. 1, 2006, pp. 170-176. doi:10.1016/j.chroma.2005.11.061

[8] V. Cornet, G. Yasmine, M. Goedele, J. Loco and J. Degroodt, "Development of a Fast Analytical Method for the Determination of Sudan Dyes in Chili- and Curry-Containing Foodstuffs by High-Performance Liquid Chromatography-Photodiode Array Detection," Journal of Agricultural and Food Chemistry, Vol. 54, No. 3, 2006, pp. 639-644. doi:10.1021/jf0517391

[9] E. Ertas, H. Ozer and C. Alasalvar, "A Rapid HPLC Method for Determination of Sudan Dyes and Para Red in Red Chilli Pepper," Food Chemistry, Vol. 105, No. 2, 2007, pp. 756-760. doi:10.1016/j.foodchem.2007.01.010

[10] L. He, Y. Su, B. Fang, X. Shen, Z. Zeng and Y. Liu, "Determination of Sudan Dye Residues in Eggs by Liquid
Chromatography and Gas Chromatography-Mass Spectrometry," Analytica Chimica Acta, Vol. 594, No. 1, 2007, pp. 139-146. doi:10.1016/j.aca.2007.05.021

[11] E. Mejia, Y. Ding, M. Mora and C. Garcia, "Determination of Banned Sudan Dyes in Chili Powder by Capillary Electrophoresis," Food Chemistry, Vol. 102, No. 4, 2007, pp. 1027-1033. doi:10.1016/j.foodchem.2006.06.038

[12] Y. Wang, D. Wei, H. Yang, Y. Yang, W. Xing, Y. Li and A. Deng, "Development of a Highly Sensitive and Specific Monoclonal Antibody-Based Enzyme-Linked Immunosorbent Assay (ELISA) for Detection of Sudan I in Food Samples," Talanta, Vol. 77, No. 5, 2009, pp. 17831789. doi:10.1016/j.talanta.2008.10.016

[13] D. Han, M. Yu, D. Knopp, R. Niessner, M. Wu and A. Deng, "Development of a Highly Sensitive and Specific Enzyme-Linked Immunosorbent Assay for Detection of Sudan I in Food Samples," Journal of Agricultural and Food Chemistry, Vol. 55, No. 16, 2007, pp. 6424-6430. doi:10.1021/jf071005j

[14] Y. Liu, Z. Song, F. Dong and L. Zhang, "Flow Injection Chemiluminescence Determination of Sudan I in Hot Chilli Sauce," Journal of Agricultural and Food Chemistry, Vol. 55, No. 3, 2007, pp. 614-617. doi:10.1021/jf063332h

[15] L. P. Wu, Y. F. Li, C. Z. Huang and Q. Zhang, "Visual Detection of Sudan Dyes Based on the Plasmon Resonance Light Scattering Signals of Silver Nanoparticles," Analytical Chemistry, Vol. 78, No. 15, 2006, pp. 5570-5577. doi:10.1021/ac0603577

[16] M. Du, X. Han, Z. Zhou and S. Wu, "Determination of Sudan I in Hot Chili Powder by Using an Activated Glassy Carbon Electrode," Food Chemistry, Vol. 105, No. 2, 2007, pp. 883-888. doi:10.1016/j.foodchem.2006.12.039

[17] M. Inagaki, K. Kaneko and T. Nishizawa, "NanocarbonsRecent Research in Japan," Carbon, Vol. 42, No. 8-9, 2004, pp. 1401-1417. doi:10.1016/j.carbon.2004.02.032

[18] Y. Yang, S. Chen, Q. Xue, A. Biris and W. Zhao, "Electron Transfer Chemistry of Octadecylamine-Functionalized Single-Walled Carbon Nanotubes," Electrochimca. Acta, Vol. 53, No. 14, 2008, p. 4936. doi:10.1016/i.electacta.2008.02.016

[19] D. Yang, L. Zhu and X. Jiang, "Electrochemical Reaction Mechanism and Determination of Sudan I at a Multi-Wall Carbon Nanotubes Modified Glassy Carbon Electrode," Journal of Electroanalytical Chemistry, Vol. 640, No. 1-2, 2010, pp. 17-22. doi:10.1016/j.jelechem.2009.12.022

[20] T. Gan, K. Li and K. Wu, "Multi-Wall Carbon NanotubeBased Electrochemical Sensor for Sensitive Determination of Sudan I," Sensors and Actuators B, Vol. 132, No. 1, 2008, pp. 134-139. doi:10.1016/j.snb.2008.01.013

[21] D. Yang, L. Zhu, X. Jiang and L. Guo, "Sensitive Determination of Sudan I at an Ordered Mesoporous Carbon Modified Glassy Carbon Electrode," Sensors and Actuators B, Vol. 141, No. 1, 2009, pp. 124-129. doi:10.1016/j.snb.2009.05.030

[22] Y. Wu, "Electrocatalysis and Sensitive Determination of Sudan I at the Singlewalled Carbon Nanotubes and Iron(III)-Porphyrin Modified Glassy Carbon Electrodes," 
Food Chemistry, Vol. 121, No. 2, 2010, pp. 580-584.

[23] H. Yin, Y. Zhou, X. Meng, T. Tang, S. Ai and L. Zhu, "Electrochemical Behaviour of Sudan I at $\mathrm{Fe}_{3} \mathrm{O}_{4}$ Nanoparticles Modified Glassy Carbon Electrode and Its Determination in Food Samples," Food Chemistry, Vol. 127, No. 3, 2011, pp. 1348-1353. doi:10.1016/j.foodchem.2011.01.097

[24] M. Zhang, A. Smith and W. Gorski, "Carbon NanotubeChitosan System for Electrochemical Sensing Based on Dehydrogenase Enzymes," Analytical Chemistry, Vol. 76, No. 17, 2004, pp. 5045-5050. doi:10.1021/ac049519u

[25] M. Zhang, C. Mullens and W. Gorski, "Coimmobilization of Dehydrogenases and Their Cofactors in Electrochemical Biosensors," Analytical Chemistry, Vol. 79, No. 6, 2007, pp. 2446-2450. doi:10.1021/ac061698n

[26] M. Zhang and W. Gorski, "Electrochemical Sensing Bas- ed on Redox Mediation at Carbon Nanotubes," Analytical Chemistry, Vol. 77, No. 13, 2007, pp. 3960-3965. doi:10.1021/ac050059u

[27] Y. Zhang and J. Zheng, "Direct Electrochemistry and Electrocatalysis of Cytochrome c Based on Chitosa-Room Temperature Ionic Liquid-Carbon Nanotubes Composite," Electrochimica Acta, Vol. 54, No. 2, 2008, pp. 749754. doi:10.1016/j.electacta.2008.06.066

[28] A. Babaei, M. Afrasiabi and M. Babazadeh. "A Glassy Carbon Electrode Modified with Multiwalled Carbon Nanotube/Chitosan Composite as a New Sensor for Simultaneous Determination of Acetaminophen and Mefenamic Acid in Pharmaceutical Preparations and Biological Samples," Electroanalysis, Vol. 22, No. 15, 2010, pp. 17431749. doi:10.1002/elan.200900578 